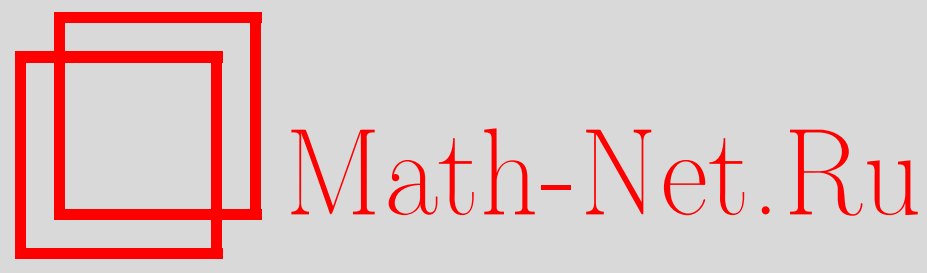

\begin{abstract}
А. А. Новиков, А. Н. Ширяев, Об одном эффективном случае решения задачи об оптимальной остановке для случайных блужданий, Теория вероятн. и ее примен., 2004, том 49, выпуск 2, 373-382
\end{abstract}

DOI: https://doi.org/10.4213/tvp227

Использование Общероссийского математического портала Math-Net.Ru подразумевает, что вы прочитали и согласны с пользовательским соглашением

http: //www.mathnet.ru/rus/agreement

Параметры загрузки:

IP : 54.224 .187 .69

26 апреля 2023 г., 14:26:56

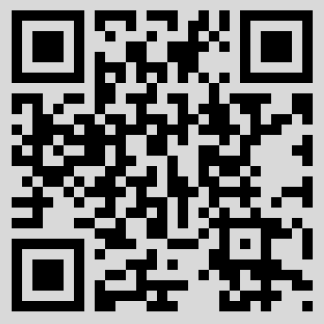


28. Егоров $B . A$. Об асимптотическом поведении самонормированных сумм случайных величин. - Теория вероятн. и ее примен., 1996, т. 41, в. 3, с. 643-650.

29. Чистлков Г. П. Новое асимптотическое разложение и асимптотически наилучшие постоянные в теореме Ляпунова. I. - Теория вероятн. и ее примен., 2001, т. 46, B. 2 , c. $326-344$.

30. Bloznelis M., Putter H. Second order and bootstrap approximation to Student's $t$ statistic. - Теория вероятн. и ее примен., 2002, т. 47, в. 2, с. 374-381.

Поступила в редакцию 15.X.2001

(C) 2004 г.

НОВИКОВ А. А. ${ }^{*}$ ШИРЯЕВ А. Н.**

\section{ОБ ОДНОМ ЭФФЕКТИВНОМ СЛУЧАЕ РЕШЕНИЯ ЗАДАЧИ ОБ ОПТИМАЛЬНОЙ ОСТАНОВКЕ ДЛЯ СЛУЧАЙНЫХ БЛУЖДАНИЙ ${ }^{1)}$}

В работе найдено решение задачи об оптимальной остановке в случае, когда функция выплат является целой степенной функцией от случайного блуждания, рассматриваемого на бесконечном временном интервале. При этом показано, что оптимальным является момент первого пересечения уровня, определяемого как наибольший корень полинома Аппеля, ассоциированного с распределением максимума случайного блуждания. Показано также, что в задаче об оптимальной остановке на конечном временном интервале $\{0,1, \ldots, T\}$ цена сходится при $T \rightarrow \infty$ с экспоненциальной скоростью к найденному пределу, когда скачки случайного блуждания экспоненциально ограничены сверху.

Ключевые слова и фразы: оптимальная остановка, случайное блуждание, скорость сходимости, полиномы Аппеля.

\section{$\S 1$. Введение и основной результат}

1. Пусть $\xi, \xi_{1}, \xi_{2}, \ldots$ - последовательность независимых одинаково распределенных случайных величин, заданных на вероятностном пространстве $(\Omega, \mathscr{F}, \mathbf{P})$. Свяжем с этой последовательностью однородную марковскую цепь $X=\left(X_{0}, X_{1}, X_{2}, \ldots\right)$ такую, что

$$
X_{0}=x \in \mathbf{R}, \quad X_{k}=x+S_{k}, \quad S_{k}=\sum_{i=1}^{k} \xi_{i}, \quad k \geqslant 1 .
$$

Через $P_{x}$ будем обозначать распределение вероятностей, отвечающее последовательности $X$. Иначе говоря, система $P_{x}, x \in \mathbf{R}$, и $X$ задают марковское семейство относительно потока $\sigma$-алгебр $\left(\mathscr{F}_{k}\right)_{k \geqslant 0}, \mathscr{F}_{0}=\{\varnothing, \Omega\}, \mathscr{F}_{k}=\sigma\left\{\xi_{1}, \ldots, \xi_{k}\right\}$.

Для рассматриваемого случайного блуждания в работе обсуждается задача об оптимальной остановке, состоящая в отыскании функции «цены»

$$
V(x)=\sup _{\tau \in \overline{\mathfrak{M}}_{0}^{\infty}} E_{x} g\left(X_{\tau}\right) I\{\tau<\infty\}, \quad x \in \mathbf{R},
$$

* Математический институт им. В. А. Стеклова РАН; Department of Mathematical Sciences, Sydney University of Technology, PO BOX 123, Broadway, Sydney, NSW 2007, Australia; e-mail: alex.novikov@uts.edu.au

** Математический институт им. В. А. Стеклова РАН, ул. Губкина, 8, 119991 Москва, ГСП-1, Россия; e-mail: albertsh@mi.ras.ru

1) Работа выполнена при поддержке ARC Large Grant A0010474; работа второго автора поддержана также Российским фондом фундаментальных исследований (грант 02-01-00834). 
где $g(x)$ - измеримая функция, $I\{\cdot\}$ - индикаторная функция и супремум берется по классу $\overline{\mathfrak{M}}_{0}^{\infty}$ всех марковских моментов $\tau$ со значениями в $[0, \infty]$ и относительно $\left(\mathscr{F}_{k}\right)_{k} \geqslant 0$. Мы называем оптимальным момент остановки $\tau^{*}$ такой, что

$$
E_{x} g\left(X_{\tau^{*}}\right) I\left\{\tau^{*}<\infty\right\}=V(x), \quad x \in \mathbf{R} .
$$

Основные предположения относительно случайного блуждания $X$ и функции $g(x)$ следуюшие:

$$
\mathbf{E} \xi<0, \quad g(x)=\left(x^{+}\right)^{n}=(\max (x, 0))^{n}, \quad n=1,2, \ldots .
$$

Хорошо известно (см., например, [4], [11]), что при весьма широких предположениях о функции $g(x)$ решение задачи (1) может быть охарактеризовано следуюшим образом: $V(x)$ есть наименьшая эксцессивная мажоранта функции $g(x)$, т.е. наименьшая из функций $U=U(x)$ со свойствами

$$
U(x) \geqslant g(x), \quad U(x) \geqslant T U(x)
$$

где $T U(x)=E_{x} U\left(X_{1}\right)=\mathbf{E} U(x+\xi)$.

При этом, опять же при весьма широких предположениях о функции $g(x)$, известно (см. [11]), что если марковский момент

$$
\tau^{*}=\inf \left\{k \geqslant 0: V\left(X_{k}\right)=g\left(X_{k}\right)\right\}
$$

является конечным $P_{x}$-п.н. при всех $x \in \mathbf{R}$, то он является оптимальньц в классе $\mathfrak{M}_{0}^{\infty}$ (марковских моментов со значениями в $[0, \infty)$ ).

В литературе известно меньше результатов относительно оптимальности момента

$$
\tau^{*}=\inf \left\{k \geqslant 0: X_{k} \in D^{*}\right\}
$$

(где «область остановки» $D^{*}=\{x \in \mathbf{R}: V(x)=g(x)\}$ и мы всегда полагаем $\inf \{\varnothing\}=\infty$ ) такого, что, вообще говоря, $P_{x}\left\{\tau^{*}=\infty\right\}>0$ для некоторых $x \in \mathbf{R}$.

В рассматриваемой нами ситуации мы как раз имеем случай, когда момент остановки не является конечнылм, но, тем не менее, оптимален (в классе $\left.\overline{\mathfrak{M}}_{0}^{\infty}\right)$. Отметим, что результаты, описанные в [4], [11], дают лишь качественное описание решения задач об оптимальной остановке для марковских семейств, но не дают эффективного способа отыскания функции $V(x)$ или построения области остановки $D^{*}$. С этой точки зрения всегда интересны те работы, в которых удается отыскать и $V(x)$, и $D^{*}$. $\mathrm{K}$ их числу можно отнести статью [6], в которой для функции $g(x)=x^{+}$в предположении $\mathbf{E} \xi<0$ устанавливается, что оптимальный момент остановки $\tau^{*} \in \overline{\mathfrak{M}}_{0}^{\infty}$ существует и имеет пороговую структуру:

$$
\tau^{*}=\inf \left\{k \geqslant 0: X_{k} \geqslant a^{*}\right\}
$$

т.е. $D^{*}=\left\{x \in \mathbf{R}: x \geqslant a^{*}\right\}$, где значение порога $a^{*}$, а также функция $V(x)$ полностью определяются по распределению величины

$$
M=\sup _{k \geqslant 0} S_{k}, \quad S_{0}=0
$$

(мы используем, по возможности, те же обозначения, что и в [6]).

2. Цель настоящей работы состоит в следующем: с одной стороны, мы обобщаем результаты работы [6] на случай функций $g(x)=\left(x^{+}\right)^{n}$ при $n=2,3, \ldots$; с другой стороны, мы предлагаем иной, нежели в [6], метод установления оптимальности соответствуюших моментов остановки (которые, как и в [6], оказываются порогового типа).

Для формулировки основного результата работы - теоремы 1 , нам потребуется следуюшее определение.

Пусть $\eta$ - случайная величина такая, что $\mathbf{E} e^{\lambda|\eta|}<\infty$ для некоторого $\lambda>0$. Определим полиномы $Q_{k}(y)=Q_{k}(y ; \eta), k=0,1,2, \ldots$, из разложения

$$
\frac{e^{u y}}{\mathbf{E} e^{u \eta}}=\sum_{k=0}^{\infty} \frac{u^{k}}{k !} Q_{k}(y) .
$$

Так определенные (по «производящей» функции $e^{u y} / \mathbf{E} e^{u \eta}$ ) полиномы $Q_{k}(y)$, $k=0,1,2, \ldots$, носят название полиномов Anпеля (их называют также полиномами 
ШефФера, см., например, [13]). Полиномы $Q_{k}(y)$ могут быть выражены через кумулянты $\varkappa_{1}, \varkappa_{2}, \ldots$ величины $\eta$. Например,

$$
\begin{gathered}
Q_{0}(x)=1, \quad Q_{1}(y)=y-\varkappa_{1}, \quad Q_{2}(y)=\left(y-\varkappa_{1}\right)^{2}-\varkappa_{2}, \\
Q_{3}(x)=\left(y-\varkappa_{1}\right)^{3}-3 \varkappa_{2}\left(y-\varkappa_{1}\right)-\varkappa_{3} .
\end{gathered}
$$

Заметим, что для однозначного определения полиномов $Q_{k}(y), k=1, \ldots, n$, достаточно, на самом деле, требовать лишь, чтобы $\mathbf{E}|\eta|^{n}<\infty$. При этом

$$
\frac{d}{d y} Q_{k}(y)=k Q_{k-1}(y), \quad k \leqslant n
$$

(это свойство иногда берется за определение полиномов Аппеля). Отметим также, что из определения (2) следует, что для всех $x \in \mathbf{R}, y \in \mathbf{R}$ и $k=1,2, \ldots$

$$
Q_{k}(y ; \eta+x)=Q_{k}(y-x ; \eta)
$$

Всюду в дальнейшем мы будем оперировать с полиномами Аппеля, порожденными случайной величиной $M=\sup _{k \geqslant 0} S_{k}$, т.е. всегда

$$
Q_{k}(y)=Q_{k}(y ; M) \text {. }
$$

Теорема 1. Пусть $n=1,2, \ldots$ биксировано. Предположим, что

$$
g(x)=\left(x^{+}\right)^{n}, \quad \mathbf{E} \xi<0, \quad \mathbf{E}\left(\xi^{+}\right)^{n+1}<\infty .
$$

Пусть $a_{n}^{*}-$ наибольший корень уравнения

$$
Q_{n}(y)=0
$$

$u$

$$
\tau_{n}^{*}=\inf \left\{k \geqslant 0: X_{k} \geqslant a_{n}^{*}\right\} .
$$

Тогда момент остановки $\tau_{n}^{*}$ является оптимальным:

$$
V_{n}(x):=\sup _{\tau \in \overline{\mathfrak{M}}_{0}^{\infty}} E_{x}\left(X_{\tau}^{+}\right)^{n} I\{\tau<\infty\}=E_{x}\left(X_{\tau_{n}^{*}}^{+}\right)^{n} I\left\{\tau_{n}^{*}<\infty\right\}
$$

при этом

$$
V_{n}(x)=\mathbf{E} Q_{n}(M+x) I\left\{M+x \geqslant a_{n}^{*}\right\} .
$$

3 а м е ч а и и е 1 . В случаях $n=1$ и $n=2$

$$
a_{1}^{*}=\mathbf{E} M, \quad a_{2}^{*}=\mathbf{E} M+\sqrt{\mathbf{D} M} .
$$

Случаи, когда распределение $M$ находится в явном виде, описаны в $[2, \S 19]$; см. также некоторые примеры в работе [14] относительно явных формул для моментов пересечения уровня полунепрерывными случайными блужданиями. В общем случае для нахождения $V_{n}(x)$, вообще говоря, нужно знать функцию распределения случайной величины $M$. Численные значения кумулянтов и функции распределения случайной величины $M$ можно найти с помошью тождества Спицера (см., например, [12]).

3 а м е ч а н и е 2. Метод доказательства теоремы 1 позволяет также получать соответствуюшие результаты и для других функций $g(x)$. В теореме 2 (см. §4 ниже) в качестве иллюстрации рассмотрен случай $g(x)=1-e^{-x^{+}}$. Отметим, что в [6] рассмотрен также случай функции $g(x)=\left(e^{x}-1\right)^{+}$(с дисконтированием по времени).

3. Идея доказательства теоремы 1 состоит в следуюшем.

Наряду с функцией $g(x)=\left(x^{+}\right)^{n}$ рассмотрим функцию $\widehat{g}(x)=x^{n}$ и решим для нее задачу об оптимальной остановке

$$
\widehat{V}_{n}(x)=\sup _{\tau \in \widehat{\mathfrak{M}}_{0}^{\infty}} E_{x} \widehat{g}\left(X_{\tau}\right) I\{\tau<\infty\}
$$

где $\widehat{\mathfrak{M}}_{0}^{\infty}-$ класс моментов остановки специального вида: $\widehat{\tau}=\tau_{a}, a \geqslant 0$, где

$$
\tau_{a}=\inf \left\{k \geqslant 0: X_{k} \geqslant a\right\} .
$$

Так как на множестве $\left\{\tau_{a}<\infty\right\}$ имеем равенство $\widehat{g}\left(X_{\tau_{a}}\right)=g\left(X_{\tau_{a}}\right)$, то, очевидно, $\widehat{V}_{n}(x) \leqslant V_{n}(x)$, поскольку $V_{n}(x)$ определяется (см. (5)) по более широкому классу моментов остановки $\overline{\mathfrak{M}}_{0}^{\infty}$, нежели в (5). 
Основываясь на свойствах полиномов Аппеля, можно дать исчерпывающее решение задачи (5): оказывается (см. §2 и §3), что

$$
\widehat{V}_{n}(x)=\mathbf{E} Q_{n}(M+x) I\left\{M+x \geqslant a_{n}^{*}\right\}
$$

и оптимальный момент $\widehat{\tau}_{n}=\tau_{a_{n}^{*}}$ (в классе $\left.\widehat{\mathfrak{M}}_{0}^{\infty}\right)$.

Далее, опять же используя свойства полиномов Аппеля, удается показать, что

$$
\widehat{V}_{n}(x) \geqslant V_{n}(x), \quad x \in \mathbf{R} .
$$

Тем самым, учитывая неравенство $\widehat{V}_{n}(x) \leqslant V_{n}(x)$, находим, что $\widehat{V}_{n}(x)=V_{n}(x)$ и что момент $\widehat{\tau}_{n}=\tau_{a_{n}^{*}}$ является оптимальным в классе $\overline{\mathfrak{M}}_{0}^{\infty}$.

Осуществление намеченного пути доказательства теоремы 1 требует ряда вспомогательных утверждений относительно свойств максимума $M=\sup _{k \geqslant 0} S_{k}$ и свойств полиномов Аппеля. Этому посвяшен $\S 2$. В 33 рассматривается вспомогательная задача об оптимальной остановке (5) и приводятся детали доказательства теоремы 1. В $\$ 4$ приводится ряд замечаний и, в частности, формулируется и доказывается теорема 2 о решении задачи об оптимальной остановке для функции $g(x)=1-e^{-x^{+}}$. Там же формулируется и доказывается теорема 3 о скорости сходимости при $T \rightarrow \infty$ функции цены задачи об оптимальной остановке на конечном интервале $\{0,1, \ldots, T\}$ для $g(x)=\left(x^{+}\right)^{n}, n=1,2, \ldots$, и $g(x)=1-e^{-x^{+}}$.

\section{§ 2. Вспомогательные результаты}

Мы всегда предполагаем ниже, что $\xi, \xi_{1}, \xi_{2}, \ldots$ - последовательность независимых одинаково распределенных случайных величин и

$$
\mathrm{E} \xi<0, \quad S_{k}=\sum_{i=1}^{k} \xi_{i}, \quad k \geqslant 1, \quad S_{0}=0, \quad M=\max _{k \geqslant 0} S_{k} .
$$

Лемма 1. Имеют место следуюшие свойства:

(a) $\mathbf{P}\{M<\infty\}=1, \mathbf{P}\{M=0\}>0 u$

$$
M \stackrel{\text { law }}{=}(M+\xi)^{+} \text {. }
$$

(b) Пусть $\sigma_{a}=\inf \left\{k \geqslant 0: S_{k} \geqslant a\right\}, a \geqslant 0, u \mathbf{E} e^{\lambda M}<\infty$ dля некоторого $\lambda \in \mathbf{R}$. Тогда при всех $u \leqslant \lambda$

$$
\mathbf{E} e^{\lambda\left(M-S_{\sigma_{a}}\right)} e^{u S_{\sigma_{a}}} I\left\{\sigma_{a}<\infty\right\}=\mathbf{E} e^{\lambda M} \mathbf{E} e^{u S_{\sigma_{a}}} I\left\{\sigma_{a}<\infty\right\} .
$$

Д ок а з а т ел ь с т в о. Свойства (а) хорошо известны - см., например, [5, раздел 10.4 , теорема 4] и $[2, \S 15]$.

Левая и правая части (10) конечны в силу предположения о конечности Е $e^{\lambda M}$. Само равенство (10) следует из того факта, что на множестве $\left\{\sigma_{a}<\infty\right\}=\{M \geqslant a\}$ выполнены неравенства $S_{k}<S_{\sigma_{a}}$ для $k<\sigma_{a}$ и, значит, имеет место равенство

$$
M-S_{\sigma_{a}}=\sup _{k \geqslant 0}\left(S_{k+\sigma_{a}}-S_{\sigma_{a}}\right) \text {. }
$$

В силу временной однородности последовательности $S_{n}$ отсюда следует, что $M-S_{\sigma_{a}} \stackrel{\text { law }}{=} M$. Заметим, кроме того, что случайная величина $M-S_{\sigma_{a}}$ на множестве $\left\{\sigma_{a}=k\right\}$ не зависит от событий из $\sigma$-алгебры $\mathscr{F}_{k}=\sigma\left\{\xi_{1}, \ldots, \xi_{k}\right\}$. Это обстоятельство и влечет свойство (10) ввиду следуюших равенств:

$$
\begin{aligned}
\mathbf{E} e^{\lambda\left(M-S_{\sigma_{a}}\right)} e^{u S_{\sigma_{a}}} I\left\{\sigma_{a}<\infty\right\} & =\mathbf{E} \sum_{k=0}^{\infty} \mathbf{E}\left(e^{\lambda\left(M-S_{k}\right)} \mid \mathscr{F}_{k}\right) e^{u S_{\sigma_{a}}} I\left\{\sigma_{a}=k\right\} \\
& =\mathbf{E} e^{\lambda M} \mathbf{E} e^{u S_{\sigma_{a}}} I\left\{\sigma_{a}<\infty\right\} .
\end{aligned}
$$

Лемма 2. (а) Пусть $\mathbf{E} e^{\lambda \xi}<1$ для некоторого $\lambda>0$. Тогда для всех $u \leqslant \lambda$

$$
\mathbf{E} e^{u M}<\infty
$$

(b) Для любого $p>0$

$$
\mathbf{E}\left(\xi^{+}\right)^{p+1}<\infty \Longrightarrow \mathbf{E} M^{p}<\infty .
$$


Д ок а з а те л ь с т в о. См. работы [8], [2], [6], а также работу [9] относительно верхних оценок для $\mathbf{P}\{M>x\}$, которые также влекут утверждение (a).

Лемма 3. Пусть $\tau_{a}=\inf \left\{k \geqslant 0: X_{k} \geqslant a\right\}, a \geqslant 0$.

(a) Eсли $\mathbf{E} e^{\lambda \xi}<1$ для некоторого $\lambda \geqslant 0$, то для всех $a \geqslant 0 u u \leqslant \lambda$

$$
E_{x} I\left\{\tau_{a}<\infty\right\} e^{u X_{\tau_{a}}}=\frac{\mathbf{E} I\{M+x \geqslant a\} e^{u(M+x)}}{\mathbf{E} e^{u M}} .
$$

(b) Ecлu $\mathbf{E}\left(\xi^{+}\right)^{n+1}<\infty$, mo $\partial \Omega s$ вcex $a \geqslant 0$

$$
E_{x} I\left\{\tau_{a}<\infty\right\} X_{\tau_{a}}^{n}=\mathbf{E} I\{M+x \geqslant a\} Q_{n}(M+x) .
$$

Д ок аз а т ельст в о. (а) По лемме 2 условие $\mathbf{E} e^{\lambda \xi}<1$ влечет конечность $\mathbf{E} e^{u M}$ для $u \leqslant \lambda$.

Если $x \geqslant a$, то $\tau_{a}=0$ и равенство (11) очевидно. При $x<a$ мы можем применить утверждение леммы 1 (b) с $\lambda=u$ :

$$
\begin{aligned}
E_{x} I\left\{\tau_{a}<\infty\right\} e^{u X_{\tau_{a}}} \mathbf{E} e^{u M} & =\mathbf{E} I\left\{\sigma_{a-x}<\infty\right\} e^{u\left(S_{\sigma_{a-x}}+x\right)} \mathbf{E} e^{u M} \\
& =\mathbf{E} I\left\{\sigma_{a-x}<\infty\right\} e^{u(M+x)}=\mathbf{E} I\{M+x \geqslant a\} e^{u(M+x)}
\end{aligned}
$$

что эквивалентно соотношению (11).

(b) Пусть выполнено условие $\mathbf{E} e^{\lambda \xi}<1$ при некотором $\lambda>0$. Тогда, очевидно, возможно дифференцирование в обеих частях (11) по параметру $u<\lambda$. Вычисляя $n$-ю производную в точке $u=0$, на основании определения полиномов Аппеля (2) получаем (12).

То, что это соотношение остается справедливым и только в предположении $\mathbf{E}\left(\xi^{+}\right)^{n+1}<\infty$, доказывается с помошью стандартного приема «усечения» скачков, состоящего в следующем.

Наряду с исходным случайным блужданием $S_{k}, k \geqslant 0$, рассмотрим случайное блуждание $S_{k}^{(N)}, k \geqslant 0$, порожденное случайной величиной $\xi^{(N)}=\min (\xi, N)$, $N=1,2, \ldots$ Далее будем использовать индекс $N$ для всех функционалов, определяемых по $S_{k}^{(N)}, k \geqslant 0$, таким же образом, как и выше для $S_{k}, k \geqslant 0: M^{(N)}=\sup _{k \geqslant 0} S_{k}^{(N)}$, $\tau_{a}^{(N)}=\inf \left\{k: X_{k}^{(N)} \geqslant a\right\}$ и т.д.

В силу леммы 2 максимум $M^{(N)}$ является экспоненциально ограниченной случайной величиной (т.е. $\mathbf{E} \exp \left\{\lambda M^{(N)}\right\}<1$ для некоторого $\lambda>0$ ), и, значит, снова по лемме 2 , соотношение (12) выполнено для «усеченного» случайного блуждания. Поэтому достаточно только проверить, что условие $\mathbf{E}\left(\xi^{+}\right)^{n+1}<\infty$ влечет, что при $N \rightarrow \infty$

и

$$
M^{(N)} \stackrel{\mathrm{d}}{\longrightarrow} M, \quad \mathbf{E}\left(M^{(N)}\right)^{k} \rightarrow \mathbf{E}(M)^{k}, \quad k=1, \ldots, n,
$$

$$
E_{x} I\left\{\tau_{a}^{(N)}<\infty\right\}\left(X_{\tau_{a}^{(N)}}^{(N)}\right)^{n} \rightarrow E_{x} I\left\{\tau_{a}<\infty\right\}\left(X_{\tau_{a}}\right)^{n} .
$$

Выполнение этих свойств непосредственно следует из интегрируемости и монотонности последовательностей $\left\{M^{(N)}\right\}$ и $\left\{X_{r_{a}^{(N)}}^{(N)}\right\}$ при $N \rightarrow \infty$.

Лемма 4. Пусть $\mathbf{E}\left(\xi^{+}\right)^{n+1}<\infty$. Тогда

$$
\mathbf{E} Q_{n}(M+x)=x^{n} \text {. }
$$

Д ок а з а т е ль с т в о. Предположим сначала, что $\mathbf{E} e^{\lambda \xi}<1$ при некотором $\lambda>0$. Тогда по лемме $2 \mathbf{E} e^{\lambda M}<\infty$, и из определения полиномов Аппеля следует, что

$$
e^{u x}=\frac{\mathbf{E} e^{u(M+x)}}{\mathbf{E} e^{u M}}=\sum_{k=0}^{\infty} \frac{u^{k}}{k !} \mathbf{E} Q_{k}(M+x), \quad 0 \leqslant u<\lambda .
$$

Отсюда легко следует (13). Общий случай доказывается с помощью уже описанного выше приема «усечения» скачков.

3 а м е ч а н и е 3 . Утверждение леммы 4 легко обобшается на случай полиномов Аппеля, порожденных произвольной случайной величиной $\eta$ такой, что $\mathbf{E}|\eta|^{n}<\infty: \mathbf{E} Q_{n}(\eta+x ; \eta)=x^{n}$. 
Лемма 5. Пусть $n=1,2, \ldots$. Тогда полином $Q_{n}(y)$ имеет единственный положительный корень $a_{n}^{*}$, причем

$$
Q_{n}(y) \leqslant 0 \quad \partial \Omega s \quad 0 \leqslant y<a_{n}^{*}
$$

$u Q_{n}(y)$ возрастает для $y \geqslant a_{n}^{*}$.

Д о к а з а т е л ь с т в о. При $n=1$ утверждение леммы выполнено, поскольку $Q_{1}(y)=y-\mathbf{E} M, a_{1}^{*}=\mathbf{E} M>0$. Дальше доказательство будет проведено по индукции на основе свойства (3). Для этого нам нужно, прежде всего, показать, что $Q_{n}(0) \leqslant 0$ при всех $n=1,2, \ldots$.

Пусть $\sigma_{a}=\inf \left\{k: S_{k} \geqslant a\right\}, a \geqslant 0$. Обозначим

$$
q(a, n):=\mathbf{E} I\left\{\tau_{a}<\infty\right\} S_{\sigma_{a}}^{n} .
$$

Очевидно, $q(a, n) \geqslant 0$ при всех $a \geqslant 0$. В силу леммы 3 (b) с $x=0$

$$
q(a, n)=\mathbf{E} I\{M \geqslant a\} Q_{n}(M) .
$$

Так как по лемме $4 \mathbf{E} Q_{n}(M)=0$, то

$$
q(a, n)=-\mathbf{E} I\{M<a\} Q_{n}(M)=-\mathbf{P}\{M<a\} Q_{n}(0)+\mathbf{E} I\{M<a\}\left(Q_{n}(0)-Q_{n}(M)\right) \text {. }
$$

Используя (3), получим

$$
\mathbf{E} I\{M<a\}\left|Q_{n}(M)-Q_{n}(0)\right| \leqslant n a \max _{0 \leqslant x \leqslant a}\left|Q_{n-1}(x)\right| \mathbf{P}\{M<a\}
$$

и поэтому

$$
q(a, n)=-\mathbf{P}\{M<a\} Q_{n}(0)+o(a) \quad \text { при } \quad a \rightarrow 0 .
$$

Так как $q(a, n) \geqslant 0$ и $\mathbf{P}\{M<a\} \geqslant \mathbf{P}\{M=0\}>0$ (см. лемму 1) при всех $a \geqslant 0$, то отсюда получаем требуемое свойство $Q_{n}(0) \leqslant 0$ для всех $n=1,2, \ldots$.

Запишем соотношение (3) в следуюшем виде:

$$
Q_{n}(y)=Q_{n}(0)+n \int_{0}^{y} Q_{n-1}(u) d u .
$$

Предполагая по индукции, что при некотором $n>1$ выполнены неравенства $Q_{n-1}(y) \leqslant 0$ для $y \in\left[0, a_{n-1}^{*}\right]$ и $Q_{n-1}(y)>0$ для $y>a_{n-1}^{*}>0$, получим, что полином $Q_{n}(y)$ отрицателен и убывает на интервале $\left(0, a_{n-1}^{*}\right)$ и достигает своего минимума в точке $y=a_{n-1}^{*}$. При $y \geqslant a_{n-1}^{*}$ полином $Q_{n}(y)$ возрастает к бесконечности, и поэтому сушествует корень $a_{n}^{*}>a_{n-1}^{*}>0$. По индукции это означает, что утверждение леммы верно для всех $n=1,2, \ldots$.

Лемма 6. Пусть

$$
f(x)=\mathbf{E} I\left\{M+x \geqslant a^{*}\right\} G(M+x)<\infty,
$$

где функиия $G(x)$ такова, что при всех $y \geqslant x \geqslant a^{*} \geqslant 0$

$$
G(y) \geqslant G(x) \geqslant G\left(a^{*}\right)=0 .
$$

Тогда для всех $x$

$$
f(x) \geqslant \mathbf{E} f(\xi+x) .
$$

Д о к а з а т е л ь с т в о. Неравенство (16) доказывает следующая цепочка соотношений, в которой используется свойство $M \stackrel{\text { law }}{=}(M+\xi)^{+}$(см. лемму 1$)$ :

$$
\begin{aligned}
f(x)= & \mathbf{E} I\left\{(M+\xi)^{+}+x \geqslant a^{*}\right\} G\left((M+\xi)^{+}+x\right)=\mathbf{E} I\left\{x \geqslant a^{*}, M+\xi<0\right\} G(x) \\
& +\mathbf{E} I\left\{M+\xi+x \geqslant a^{*}, M+\xi \geqslant 0\right\} G(M+\xi+x) \\
\geqslant & \mathbf{E} I\left\{M+\xi+x \geqslant a^{*}, M+\xi<0\right\} G(M+\xi+x) \\
& +\mathbf{E} I\left\{M+\xi+x \geqslant a^{*}, M+\xi \geqslant 0\right\} G(M+\xi+x)=\mathbf{E} f(x+\xi) .
\end{aligned}
$$
вcex $x$

Лемма 7. Пусть $f(x)$ и $g(x)$ - неотричательные функции такие, что для

$$
u
$$$$
f(x) \geqslant g(x)
$$

Тогда для всех $x$

$$
f(x) \geqslant \mathbf{E} f(\xi+x)
$$

$$
f(x) \geqslant \sup _{\tau \in \overline{\mathfrak{M}}_{0}^{\infty}} \mathbf{E} I\{\tau<\infty\} g\left(S_{\tau}+x\right) .
$$


Д о к а з а т е л ь с т в о. Условия (17) и (18) означают, что функция $f(x)$ есть эксцессивная мажоранта для функции $g(x)$, для которой приведенное неравенство следует из теоремы Дуба о сохранении супермартингального свойства при случайной замене времени (см., например, [1], [3], [9]).

\section{§3. Доказательство теоремы 1}

Пусть $g(x)=\left(x^{+}\right)^{n}, \widehat{g}(x)=x^{n}$ и функция $\widehat{V}_{n}(x)$ определена в (5). Покажем, что выполнено (8), т.е.

$$
\widehat{V}_{n}(x)=\sup _{a \geqslant 0} E_{x} I\left\{\tau_{a}<\infty\right\} X_{\tau_{a}}^{n}=\mathbf{E} Q_{n}(M+x) I\left\{M+x \geqslant a_{n}^{*}\right\} .
$$

Для этого заметим, что по лемме $3(\mathrm{~b})$

$$
E_{x} I\left\{\tau_{a}<\infty\right\} X_{\tau_{a}}^{n}=\mathbf{E} Q_{n}(M+x) I\{M+x \geqslant a\}
$$

где $Q_{n}(M+x) \geqslant 0$ на множестве $\{M+x \geqslant a\}$ для всех $a \in\left[a_{n}^{*}, \infty\right)$. Следовательно, $\mathbf{E} Q_{n}(M+x) I\{M+x \geqslant a\}$ является убывающей функцией на $\left[a_{n}^{*}, \infty\right)$.

Пусть теперь $a \in\left[0, a_{n}^{*}\right]$. Из (13) находим, что

$$
\begin{aligned}
\mathbf{E} Q_{n}(M+x) I\{M+x \geqslant a\}= & x^{n}-\mathbf{E} Q_{n}(M+x) I\{M+x<0\} \\
& -\mathbf{E} Q_{n}(M+x) I\{0 \leqslant M+x<a\} .
\end{aligned}
$$

Пользуясь тем, что $Q_{n}(M+x) I\{0 \leqslant M+x \leqslant a\} \leqslant 0$ (см. лемму 5) и, значит, $\mathrm{E} Q_{n}(M+x) I\{0 \leqslant M+x<a\}$ является убывающей функцией, заключаем, что $\mathbf{E} Q_{n}(M+x) I\{M+x \geqslant a\}$ является возрастающей функцией на $\left[0, a_{n}^{*}\right]$. Так как эта функция является также и убывающей на $\left[a_{n}^{*}, \infty\right)$ (это показано выше) и, очевидно, непрерывной (по свойству интеграла Лебега) при всех $a$, то она достигает максимума в точке $a=a_{n}^{*}$. Таким образом, (20) (оно же (8)) доказано.

Чтобы завершить доказательство, нам нужно только проверить неравенство (8), т.е. показать, что $\widehat{V}_{n}(x) \geqslant V_{n}(x)$ (как отмечалось в $\S 1$, противоположное неравенство очевидно). С этой целью рассмотрим функцию

$$
f(x)=\widehat{V}_{n}(x)=\mathbf{E} I\left\{M+x \geqslant a_{n}^{*}\right\} Q_{n}(M+x)
$$

и применим лемму 7, беря $g(x)=\left(x^{+}\right)^{n}$. Проверим сначала условие (17) при $x \in\left(0, a_{n}^{*}\right)$ (иначе оно очевидно). Для этого заметим, что при $x \in\left(0, a_{n}^{*}\right)$ ввиду леммы 5

$$
I\left\{M+x \geqslant a_{n}^{*}\right\} Q_{n}(M+x)=\left(Q_{n}(M+x)\right)^{+} .
$$

Тогда по неравенству Иенсена и лемме 4

$$
f(x)=\mathbf{E}\left(Q_{n}(M+x)\right)^{+} \geqslant\left(\mathbf{E} Q_{n}(M+x)\right)^{+}=\left(x^{+}\right)^{n}=g(x) .
$$

Условие (18) леммы 7 выполнено для функции $G(y)=Q_{n}(y)$ в силу леммы 6 .

Таким образом, функция $f(x)$ является эксцессивной мажорантой функции $g(x)=\left(x^{+}\right)^{n}$ и, значит, $f(x) \geqslant V_{n}(x)$. Но $f(x)=\widehat{V}_{n}(x)$, и, следовательно, $\widehat{V}_{n}(x) \geqslant V_{n}(x)$. Теорема 1 доказана.

\section{§4. Несколько замечаний}

3 а м е ч а н и е 4. Метод доказательства теоремы 1 может быть использован и для других функций $g(x)$. Для примера приведем следующий результат.

Теорема 2. Пусть $\mathbf{E} \xi<0 u g(x)=1-e^{-x^{+}}$. Положим

$$
a^{*}=-\ln \mathbf{E} e^{-M} \text {. }
$$

Тогда момент остановки

$$
\tau_{a^{*}}=\inf \left\{k \geqslant 0: X_{k} \geqslant a^{*}\right\}
$$

является оптимальным:

$$
V(x)=\sup _{\tau \in \overline{\mathfrak{M}}_{0}^{\infty}} E_{x} g\left(X_{\tau}\right) I\{\tau<\infty\}=E_{x} g\left(X_{\tau_{a^{*}}}\right) I\left\{\tau_{a^{*}}<\infty\right\} ;
$$

$n p u$ этом $V(x)=\mathbf{E}\left(1-e^{-M-x}\left(\mathbf{E} e^{-M}\right)^{-1}\right)^{+}$. 
Д о к а з а т е л ь с т в о. Мы наметим только схему доказательства, поскольку оно аналогично доказательству теоремы 1 (и даже проще).

Пусть $g(x)=1-e^{-x^{+}}$и

$$
\widehat{V}(x)=\sup _{a \geqslant 0} E_{x} I\left\{\tau_{a}<\infty\right\} g\left(X_{\tau_{a}}^{n}\right) .
$$

Покажем, что

$$
\widehat{V}(x)=\mathbf{E}\left(1-\frac{e^{-M-x}}{\mathbf{E} e^{-M}}\right)^{+} .
$$

Для этого заметим, что по лемме 3 (а) с $u=-1$ справедливо равенство

и, значит,

$$
E_{x} I\left\{\tau_{a}<\infty\right\} e^{-X_{\tau_{a}}}=\frac{\mathbf{E} I\{M+x \geqslant a\} e^{-(M+x)}}{\mathbf{E} e^{-M}},
$$

$$
q(a):=E_{x} I\left\{\tau_{a}<\infty\right\} g\left(X_{\tau_{a}}\right)=\mathbf{E} I\{M+x \geqslant a\}\left(1-\frac{e^{-(M+x)}}{\mathbf{E} e^{-M}}\right) .
$$

Заметим также, что функция $1-e^{-a} / \mathrm{E} e^{-M}$ является монотонной по $a$ с единственным корнем $a^{*}=-\ln \mathrm{E} e^{-M}$. Те же рассуждения, что и в доказательстве теоремы 1 , показывают, что $q(a)$ достигает максимума в точке $a=a^{*}$ и при этом

$$
\widehat{V}(x)=\mathbf{E} I\left\{M+x \geqslant a^{*}\right\}\left(1-\frac{e^{-(M+x)}}{\mathbf{E} e^{-M}}\right)=\mathbf{E}\left(1-\frac{e^{-M-x}}{\mathbf{E} e^{-M}}\right)^{+} .
$$

Чтобы завершить доказательство, нам нужно только проверить неравенство (8), т.е. показать, что $\widehat{V}(x) \geqslant V(x)$. С этой целью рассмотрим функцию

$$
f(x)=\widehat{V}(x)=\mathbf{E}\left(1-\frac{e^{-M-x}}{\mathbf{E} e^{-M}}\right)^{+} .
$$

Условие (17) выполнено по неравенству Иенсена:

$$
f(x) \geqslant\left(1-\frac{\mathbf{E} e^{-M-x}}{\mathbf{E} e^{-M}}\right)^{+}=\left(1-e^{-x}\right)^{+}=g(x) .
$$

Условие (18) выполнено для функции $G(y)=\left(1-e^{-y} / \mathbf{E} e^{-M}\right)^{+}$в силу леммы 6 . Все это приводит к доказательству теоремы 2.

3 а м е ч а н и е 5 . Решение задачи об оптимальной остановке на конечном интервале $\{0,1, \ldots, T\}$, состояшей в отыскании «цены»

$$
V(x, T)=\sup _{\tau \in \mathfrak{M}_{0}^{T}} E_{x} g\left(X_{\tau}\right), \quad x \in \mathbf{R}
$$

где супремум берется по всем марковским моментам $\tau, \tau \leqslant T<\infty$, можно получить в численном виде, используя, как хорошо известно, метод «обратной индукции» (детали см., например, в [4], [11]). При больших $T$ реализация этого метода может потребовать большого счета даже для простых распределений. Поэтому представляет интерес оценить скорость сходимости $V(x, T)$ при $T \rightarrow \infty$ к функции $V(x)$, найденной в теоремах 1 и 2 .

Теорема 3. Пусть $g(x)=\left(x^{+}\right)^{n}, n=1,2, \ldots$, или $g(x)=1-e^{-x^{+}}, u$ пусть $\mathbf{E} e^{\lambda \xi}<1$ для некоторого $\lambda>0$. Тогда существуют положительные константы $C(x)$ и с, не зависяиие от $T$ и такие, ито для каждого $x \in \mathbf{R}$ при всех $T>0$

$$
0 \leqslant V(x)-V(x, T) \leqslant C(x) e^{-c T} .
$$

Д о к а з а т е л ь с т в о. Так как класс $\mathfrak{M}_{0}^{\infty}$ шире класса $\mathfrak{M}_{0}^{T}$, то $V(x) \geqslant$ $V(x, T)$. Чтобы получить верхнюю оценку (23), заметим, что $V(x, T) \geqslant$ $E_{x} g\left(X_{\min \left(\tau_{b}, T\right)}\right) \geqslant E_{x} g\left(X_{\tau_{b}}\right) I\left\{\tau_{b} \leqslant T\right\}$, где $\tau_{b}=\inf \left\{k \geqslant 0: X_{k} \geqslant b\right\}$ - оптимальный момент остановки для цены $V(x)$ и, согласно теоремам 1 или 2 , параметр $b$ 
равен $a_{n}^{*}$ или $a^{*}-$ соответственно виду рассматриваемой функции $g(x)$. Так как $V(x)=E_{x} g\left(X_{\tau_{b}}\right) I\left\{\tau_{b}<\infty\right\}$, то получаем

$$
V(x)-V(x, T) \leqslant E_{x} g\left(X_{\tau_{b}}\right) I\left\{T<\tau_{b}<\infty\right\} .
$$

Ниже мы покажем, что

$$
P_{x}\left\{T<\tau_{b}<\infty\right\} \leqslant C(x) e^{-c T},
$$

где $C(x)$ и $c$ - некоторые положительные константы, не зависяшие от $T$. Если функция $g(x)$ ограничена (как в теореме 2), то отсюда и из (24) сразу следует (23). Этой же оценки (25) достаточно для справедливости утверждения (23) и в случае $g(x)=\left(x^{+}\right)^{n}$, $n=1,2, \ldots$, в силу неравенства $X_{\tau_{b}} \leqslant M$ в комбинации с леммой $2(\mathrm{~b})$ и неравенством Гёльдера.

Пусть $\psi(u)$ определяется из соотношения

$$
\mathbf{E} e^{u \xi}=e^{\psi(u)} \quad(u<\theta=\sup \{u \geqslant 0: \psi(u)<\infty\}) .
$$

Заметим, что $\psi(u)$ - выпуклая функция и в силу условий теоремы $\theta>0$. Из этих свойств следует, что производная $\psi^{\prime}(0)=\mathbf{E} \xi<0$ и существует положительное $\lambda_{0} \in(0, \theta)$ такое, что $\psi^{\prime}\left(\lambda_{0}\right)=0$ и $\psi\left(\lambda_{0}\right)<0$ (см., например, [2]). Чтобы теперь доказать (25), воспользуемся тем фактом, что процесс

$$
\exp \left\{\lambda\left(X_{k}-x\right)-k \psi(\lambda)\right\}, \quad k \geqslant 0 \quad(\lambda \in(0, \theta))
$$

является неотрицательным мартингалом с математическим ожиданием $\mathbf{E} \exp \left\{\lambda\left(X_{k}-\right.\right.$ $x)-k \psi(\lambda)\}=1$ и поэтому может быть использован для задания новой меры $\widehat{P}_{x}(A)$, $A \in \sigma\left(\bigcup_{k \geqslant 0} \mathscr{F}_{k}\right)$ такой, что для каждого $k \geqslant 0$ и множества $A \in \mathscr{F}_{k}$

$$
\widehat{P}_{x}(A)=E_{x} I\{A\} \exp \left\{\lambda_{0}\left(X_{k}-x\right)-k \psi\left(\lambda_{0}\right)\right\} .
$$

(Это есть не что иное, как общеизвестное преобразование Эшера.) Из данных определений следует, что при $\lambda_{0} \in(0, \theta)$

$$
\begin{aligned}
& E_{x} I\left\{T<\tau_{b}<\infty\right\} \exp \left\{\lambda_{0}\left(X_{\tau_{b}}-x\right)-\tau_{b} \psi\left(\lambda_{0}\right)\right\} \\
& =\sum_{k=T+1}^{\infty} E_{x} I\left\{\tau_{b}=k\right\} \exp \left\{\lambda_{0}\left(X_{k}-x\right)-k \psi\left(\lambda_{0}\right)\right\} \\
& \quad=\sum_{k=T+1}^{\infty} \widehat{P}_{x}\left\{\tau_{b}=k\right\}=\widehat{P}_{x}\left\{T<\tau_{b}<\infty\right\} .
\end{aligned}
$$

Так как $X_{\tau_{b}} \geqslant b$, то

$$
P_{x}\left\{T<\tau_{b}<\infty\right\} \leqslant e^{\lambda_{0}(x-b)} e^{\psi\left(\lambda_{0}\right) T} \widehat{P}_{x}\left\{T<\tau_{b}<\infty\right\} .
$$

Ввиду того, что $\widehat{P}_{x}\left\{T<\tau_{b}<\infty\right\} \leqslant 1$, отсюда следует оценка (25) с константами $c=-\psi\left(\lambda_{0}\right)>0, C(x)=e^{\lambda_{0}(x-b)}, b=a_{n}^{*}$ или $b=a^{*}$ соответственно виду рассматриваемой функции $g(x)$.

Теорема 3 доказана.

3 а м е ч а н и е 6 . Результаты работы непосредственным образом переносятся на случай непрерывного времени, когда вместо случайного блуждания $S_{n}$ рассматривается однородный процесс Леви. Этот перенос можно сделать либо с помощью предельного перехода от дискретного времени по аналогии с тем, как это сделано в [10] применительно к задаче об Американском опционе, либо с помощью техники псевдодифференциальных операторов в контексте процессов Леви, описанной в монографии [3].

Авторы признательны Е. Шинджикашвили за обсуждение результатов работы. Первый автор также признателен Y. Miyahara и A. Shimizu за поддержку и дискуссии во время визита в Nagoya City University. 


\section{СПИСОК ЛИТЕРАТУРЫ}

1. Blackwell D. On optimal systems. - Ann. Math. Statist., 1954, v. 25, p. 394-397.

2. Боровков A. A. Вероятностные процессы в теории массового обслуживания. М.: Наука, 1972, 368 c.

3. Boyarchenko S., Levendorski乞 S.Z. Non-Gaussian Merton-Black-Scholes Theory. River Edge: World Scientific, 2002, 398 p. (Adv. Ser. Statist. Sci. Appl. Probab., v. 9.)

4. Роббинс Г., Сигмунд’ Д., Чао И. Теория оптимальных правил остановки. М.: Наука, 1977, 167 с.

5. Chow Y.S., Teicher T. Probability Theory: Independence, Interchangeability, Martingales. New York: Springer-Verlag, 1997, $488 \mathrm{p}$.

6. Darling D. A., Liggett T., Taylor H.M. Optimal stopping for partial sums. - Ann. Math. Statist., 1972, v. 43, p. 1363-1368.

7. Dubins L. E., Savage L. J. Inequalities for Stochastic Processes (How to Gamble if You Must). New York: Dover, 1976, 255 p.

8. Kingman J. F. C. Inequalities in the theory of queues. - J. Roy. Statist. Soc. Ser. B, 1970 , v. 32 , p. $102-110$.

9. Liu Z., Nain P., Towsley D. Bounds for a class of stochastic recursive equations. Math. Methods Oper. Res., 1999, v. 49, № 2, p. 325-333.

10. Mordecki E. Optimal stopping and perpetual options for Lévy processes. - Finance Stoch., 2002, v. 6, № 4, p. 473-493.

11. Ширяев А.Н. Статистический последовательный анализ. Оптимальные правила остановки. М.: Наука, 1976, 272 с.

12. Stadje $W$. An iterative approximation procedure for the distribution of the maximum of a random walk. - Statist. Probab. Lett., 2000, v. 50, № 4, p. 375-381.

13. Schoutens $W$. Stochastic Processes and Orthogonal Polynomials. New York: SpringerVerlag, 2000, 163 p. (Lecture Notes in Statist., v. 146.)

14. Висков $О$. В. Случайное блуждание с непрерывной вверх компонентой и формула обрашения Лагранжа. - Теория вероятн. и ее примен., 2000, т. 45, в. 1, с. 166-175.

Поступила в редакцию

1.VII.2002

\section{() 2004 г. САХАНЕНКО А. И.*}

\section{О ПЕРЕХОДНЫХ ЯВЛЕНИЯХ В СЛУЧАЙНЫХ БЛУЖДАНИЯХ}

Пусть $\bar{S}_{n}=\max _{1 \leqslant k \leqslant n} \sum_{i=1}^{k} X_{i, n}$, где при каждом $n=1,2, \ldots$ последовательность $X_{1, n}, \ldots, X_{n, n}$ состоит из независимых и одинаково распределенных случайных величин с конечными положительными дисперсиями. В работе изучается задача о получении простых и неулучшаемых достаточных условий, типа условия Линдеберга, которые гарантировали бы сходимость нормированной величины $\left(\bar{S}_{n}-A_{n}\right) / B_{n}$ к некоторой невырожденной случайной величине при соответствуюшим образом подобранных постоянных $A_{n}$ и $B_{n}>0$. Упрошены, уточнены и усилены результаты, полученные ранее в этом направлении Ю. В. Прохоровым и А.А. Боровковым. В частности, подробно рассмотрен неисследованный ранее случай, когда $\mathbf{D} X_{1, n} \rightarrow 0$ при $n \rightarrow \infty$.

Ключевые слова и фразы: схема серий, максимум последовательных сумм, равномерная сходимость распределений, предельные распределения, принцип инвариантности, расстояние Прохорова.

* Югорский государственный университет, ул. Чехова, 16, 628012 ХантыМансийск, Россия; e-mail: aisakh@hotmail.com 